\title{
THE GENUS OF AN ABSTRACT INTERSECTION SEQUENCE
}

\author{
PETER PERCELL
}

\begin{abstract}
An intersection sequence, denoted $I S$, is a combinatorial object associated with a normal immersion $f: S^{1} \rightarrow M$ where $S^{1}$ is an oriented circle and $M$ is a closed, connected, oriented 2-manifold. The genus of $I S$, denoted $\gamma(I S)$, is defined to be the smallest number which is the genus of a manifold $M$ admitting a realization $g: S^{1} \rightarrow M$ of $I S$. A method is given for computing $\gamma(I S)$ from $I S$.
\end{abstract}

Throughout $S^{1}$ denotes a smooth circle oriented by a nonvanishing vector field $\theta$ and $M$ denotes a smooth, closed, connected, orientable 2-manifold oriented by a nonvanishing smooth 2 -form $\omega$. All maps between manifolds are $C^{1}$. An immersion $f: S^{1} \rightarrow M$ is called a normal immersion if

(a) $f^{-1}(y)$ contains at most 2 points for every $y \in M$, and

(b) $f^{-1}(y)=\left\{x_{1}, x_{2}\right\}$ implies $f^{\prime}\left(x_{1}\right)$ and $f^{\prime}\left(x_{2}\right)$ are linearly independent (where $f^{\prime}(x)=f_{*}(\theta(x))$ and $f_{*}$ is the differential map of $f$ ).

It follows easily from the definition that a normal immersion has only finitely many double points, i.e. points $y \in M$ such that $f^{-1}(y)$ contains 2 points.

An abstract intersection sequence $I S$ is a triple $\{n, \sigma, \nu\}$ consisting of

(a) a positive integer $n$,

(b) a bijection $\sigma: I_{n} \rightarrow I_{n}$, where $I_{n}=\{ \pm 1, \ldots, \pm n\}$, such that for all $i, j$ $\in I_{n}$ there is a positive integer $k$ such that $\sigma^{k}(i)=j$ (where $\sigma^{k}$ is composition of $\sigma$ with itself $k$ times) -this is just a cyclic ordering of $I_{n}$, and

(c) a map $\nu:\{1, \ldots, n\} \rightarrow\{ \pm 1\}$.

If $f$ is a normal immersion with $n$ double points a labeling $L$ of $f$ is a naming as $y_{1}, \ldots, y_{n}$ of the double points in $M$ and a naming of their preimages

$$
f^{-1}\left(y_{i}\right)=\left\{x_{-i}, x_{i}\right\}, \quad i=1, \ldots, n \text {. }
$$

Given a labeling $L$ of the normal immersion $f$, the intersection sequence $I S(f, L)$ is the abstract intersection sequence $\{n, \sigma, \nu\}$ determined as follows:

(a) $n$ is the number of double points of $f$,

(b) for each $j \in I_{n}, \sigma(j)$ is the $k \in I_{n}$ such that $x_{k}$ is the first $x_{i}$ encountered when moving away from $x_{j}$ in the positive direction on $S^{1}$, and

(c) $\nu(j)$ is the orientation of the crossing at $y_{j}$ defined by

Received by the editors January $15,1975$.

AMS (MOS) subject classifications (1970). Primary 55A99, 55C99, 57D40; Secondary 05C10.

Key words and phrases. Normal immersion, intersection sequence, realization, genus, tubular neighborhood. 


$$
\nu(j)=\operatorname{sgn}\left(\omega\left(f^{\prime}\left(x_{-j}\right), f^{\prime}\left(x_{j}\right)\right)\right) .
$$

A normal immersion $g: S^{1} \rightarrow M$ is called a realization of an abstract intersection sequence $I S$ if there is a labeling $L$ of $g$ such that $I S(g, L)=I S$.

Note that our definition of intersection sequences differs slightly, but not essentially, from earlier definitions. There is no need for a base point or starting point for $S^{1}$ when the target is a closed 2-manifold rather than the plane. Also, this definition eliminates the usual redundancy in the signing function $\nu$. See Francis [1] for a list of references and a short historical review of intersection sequences and of the problem of finding conditions on IS which are necessary and sufficient for $\gamma(I S)$ to be 0 . Also see Marx [3]. The problem of computing $\gamma(I S)$ generally, which we will now solve, was posed by Francis [1].

Given an abstract intersection sequence $I S=\{n, \sigma, \nu\}$ we construct a space $T(I S)$, our main tool, called the abstract tubular neighborhood associated with IS (compare with Francis [2]) as follows: Provide $S^{1}$ with a Riemannian metric so that the total length of $S^{1}$ is $2 n$. Pick any point in $S^{1}$, label it $x_{1}$ and then move in the positive direction from $x_{1}$ labeling the point a distance $k-1$ from $x_{1}$ with the name $x_{\sigma^{k}(1)}$. A set of $2 n$ equally spaced points around $S^{1}$ is obtained and these points are labeled with the names $x_{ \pm 1}, \ldots, x_{ \pm n}$ and are in the cyclic order determined by $\sigma$. Let $e>0$ be a real number small enough so that the closed arcs $\left[x_{i}-e, x_{i}+e\right]$ of $S^{1}$ (an abuse of notation with the obvious meaning) are pairwise disjoint for $i \in I_{n}$. Consider the space $[-e, e]$ $\times S^{1}$. It is a smooth 2-manifold with boundary which can be oriented by letting the ordered pair of tangent vectors $(d / d t, \theta)$, where $t$ is the coordinate for the interval of reals $[-e, e]$, determine the positive orientation at each point. Let $T(I S)$ be the space obtained from $[-e, e] \times S^{1}$ by identifying the point $\left(t, x_{-j}+s\right)$ with the point $\left(\nu(j) s, x_{j}-\nu(j) t\right)$ for all $t, s \in[-e, e]$. The idea is that according to whether $\nu(j)$ is positive or negative we rotate the square $[-e, e] \times\left[x_{-j}-e, x_{-j}+e\right]$ clockwise or counterclockwise through $90^{\circ}$ and then identify it with the square $[-e, e] \times\left[x_{j}-e, x_{j}+e\right]$. It is easily seen that $T(I S)$ is an orientable manifold whose boundary is a collection of piecewise smooth circles. Let $T:[-e, e] \times S^{1} \rightarrow T(I S)$ be the identification map. Then $T$ is an immersion and we can orient $T(I S)$ by taking $T$ to be orientation preserving.

Let $M(I S)$ be the smooth, closed, oriented, 2-manifold obtained from $T(I S)$ by smoothing its boundary circles and capping off the smoothed circles with 2-disks. Let $b(I S)$ be the number of circles in the boundary of $T(I S)$.

THEOREM 1. If IS is an arbitrary abstract intersection sequence, then IS is realizable and

$$
\gamma(I S)=\frac{1}{2}(n+2-b(I S))
$$

which is the genus of $M(I S)$.

Proof. By construction, $T \mid\{0\} \times S^{1}: S^{1} \rightarrow M(I S)$ is a realization of $I S$.

$M(I S)$ has a cell decomposition with $n$ 0-cells, $2 n$ 1-cells and $b(I S)$ 2-cells so its Euler characteristic is $b(I S)-n$. Since the genus $g$ of $M(I S)$ is related 
to its Euler characteristic $h$ by the formula $\chi=2-2 g$, it follows that $\frac{1}{2}(n+2-b(I S))$ is the genus of $M(I S)$.

Now, let $f: S^{1} \rightarrow M$ be an arbitrary realization of $I S$. It is not hard to modify the Francis [2] construction of normal tubular neighborhoods to show that $f\left(S^{1}\right)$ has a neighborhood $U$ in $M$ which is diffeomorphic to $T(I S)$. Then the closure of $M-U$ in $M$ is an orientable compact 2-manifold whose boundary is a union of piecewise smooth circles. If this complementary 2manifold is not a disjoint union of 2-disks, then it contains or creates handles which make the genus of $M$ larger than the genus of $M(I S)$. Thus $\gamma(I S)$ is the genus of $M(I S)$.

Implicit in the argument above is a uniqueness result for minimum genus realizations. In fact, we easily obtain a generalization of results of Treybig [4] and Verhey [5]. Two maps $f_{i}: S^{1} \rightarrow M_{i}, i=1,2$, are said to be equivalent if there exist orientation preserving diffeomorphisms $\alpha$ of $S^{1}$ and $\beta: M_{1} \rightarrow M_{2}$ such that $f_{2}=\beta \circ f_{1} \circ \alpha$.

THEOREM 2. Let $f_{i}: S^{1} \rightarrow M_{i}, i=1,2$, be two realizations of the abstract intersection sequence IS. If the genus of both $M_{1}$ and $M_{2}$ is $\gamma(I S)$, then $f_{1}$ and $f_{2}$ are equivalent.

SKETCH OF PROOF. The diffeomorphism $\beta$ is easily constructed to take the image $f_{1}\left(S^{1}\right)$ to the image $f_{2}\left(S^{1}\right)$ by noting that these images both have tubular neighborhoods diffeomorphic to $T(I S)$ and that these diffeomorphic tubular neighborhoods have diffeomorphic complements. Then $\alpha$ is produced by noting that $f_{2}$ and $\beta \circ f_{1}$ describe the same oriented curve in $M_{2}$ so since they are both immersions they differ by an orientation preserving reparameterization of $S^{1}$.

We finish this note by showing how to compute the number $b(I S)$ which by Theorem 1 immediately gives $\gamma(I S)$. Let

$$
E_{n}=\left\{\left(t, x_{i}+s\right) \mid t, s= \pm e \text { and } i \in I_{n}\right\} .
$$

$E_{n}$ is just the set of points in $[-e, e] \times S^{1}$ which correspond to endpoints of maximal smooth arcs in the boundary of $T(I S)$. Let an equivalence relation $\sim$ on $E_{n}$ be generated by the following equivalences:

(a) $\left(t, x_{-j}+s\right) \sim\left(\nu(j) s, x_{j}-\nu(j) t\right), t, s= \pm e$, and

(b) $\left(t, x_{k}+e\right) \sim\left(t, x_{\sigma(k)}-e\right), t= \pm e, k \in I_{n}$.

THEOREM 3. The number $b(I S)$ is the number of equivalence classes of the equivalence relation $\sim$ on $E_{n}$.

Proof. By (a) points of $E_{n}$ are equivalent if they represent the same point in $T(I S)$ and by (b) two points of $E_{n}$ are equivalent if they correspond to the two endpoints of a single smooth arc in the boundary of $T(I S)$. It follows immediately that two points of $E_{n}$ are equivalent under $\sim$ iff they project to points which are on the same component of the boundary of $T(I S)$, so there is a one-to-one correspondence between equivalence classes in $E_{n}$ and boundary components of $T(I S)$.

\section{REFERENCES}

1. G. K. Francis, Null genus realizability criterion for abstract intersection sequences, J. 
Combinatorial Theory 7 (1969), 331-341. MR 40 \#475.

2. - The folded ribbon theorem. A contribution to the study of immersed circles, Trans. Amer. Math. Soc. 141 (1969), 271-303. MR 39 \# 4863.

3. M. L. Marx, The Gauss realizability problem, Proc. Amer. Math. Soc. 22 (1969), 610-613. MR 39 \#6297.

4. L. B. Treybig, Prime mappings, Trans. Amer. Math. Soc. 130 (1968), 248-253. MR 36 \# 879.

5. R. F. Verhey, Diffeomorphic invariants of immersed circles, Trans. Amer. Math. Soc. 163 (1972), 47-63. MR 44 \#3336.

Department of Mathematics, University of Chicago, Chicago, Illinois 60637 